\title{
Can the concept of Health Promoting Schools help to improve students' health knowledge and practices to combat the challenge of communicable diseases: Case study in Hong Kong?
}

\author{
Albert Lee*1,2, Martin CS Wong2', Vera MW Keung1, Hilda SK Yuen', \\ Frances Cheng ${ }^{1}$ and Jennifer SY Mok ${ }^{1}$
}

Address: ${ }^{1}$ Centre for Health Education and Health Promotion, The Chinese University of Hong Kong, Hong Kong, China and ${ }^{2}$ Department of Community and Family Medicine, The Chinese University of Hong Kong, Hong Kong, China

Email: Albert Lee* - alee@cuhk.edu.hk; Martin CS Wong - martin_wong@cuhk.edu.hk; Vera MW Keung - verakeung@cuhk.edu.hk; Hilda SK Yuen - hildayuen@cuhk.edu.hk; Frances Cheng - fcheng@cuhk.edu.hk; Jennifer SY Mok - jenmok@gmail.com

* Corresponding author

Published: 30 January 2008

BMC Public Health 2008, 8:42 doi:10.1 186/147/-2458-8-42
Received: II February 2007

Accepted: 30 January 2008

This article is available from: http://www.biomedcentral.com/I47I-2458/8/42

(c) 2008 Lee et al; licensee BioMed Central Ltd.

This is an Open Access article distributed under the terms of the Creative Commons Attribution License (http://creativecommons.org/licenses/by/2.0), which permits unrestricted use, distribution, and reproduction in any medium, provided the original work is properly cited.

\begin{abstract}
Background: The growing epidemics of emerging infectious diseases has raised the importance of a setting approach and include the Health Promoting School (HPS) framework to promote better health and hygiene. Built on the concept of 'the' HPS framework, the Hong Kong Healthy Schools Award scheme includes "Personal Health Skills" as one of its key aspects to improve student hygiene knowledge and practices. This study examines the differences in student perceptions, knowledge and health behaviours between those schools that have adopted the HPS framework and those that have not adopted.
\end{abstract}

Methods: A cross-sectional study using multi-stage random sampling was conducted among schools with awards (HSA) and those schools not involved in the award scheme nor adopting the concept of HPS (non-HPS). For HSA group, 5 primary schools and 7 secondary schools entered the study with 510 students and 789 students sampled respectively. For the 'Non-HPS' group, 8 primary schools and 7 secondary schools entered the study with 676 students and 725 students sampled respectively. A self-administered questionnaire was used as the measuring instrument.

Results: Students in the HSA category were found to be better with statistical significance in personal hygiene practice, knowledge on health and hygiene, as well as access to health information. HSA schools were reported to have better school health policy, higher degrees of community participation, and better hygienic environment.

Conclusion: Students in schools that had adopted the HPS framework had a more positive health behaviour profile than those in non-HPS schools. Although a causal relationship is yet to be established, the HPS appears to be a viable approach for addressing communicable diseases.

\section{Background}

Health behaviours are strongly determined by the differ- ent social, economic and environmental circumstances of individuals and populations. Improvement of health lit- 
eracy can help individuals to tackle the determinants of health better as it builds up the personal, cognitive and social skills which determine the ability of individuals to gain access to, understand and use of information to promote and maintain good health [1]. Schools are essential in helping students to achieve health literacy [2]. The concept of the health promoting school (HPS) has been advocated as an effective approach to promote health in schools [3,4]. It embodies a holistic, whole school approach in which a broad health education curriculum is supported by the environment and ethos of the school and shifts health into a more dynamic and political domain to address the determinants of heath $[2,5]$. Healthy Schools Award schemes are very popularamong the European countries as structured frameworks acting as systems for monitoring and recognition of achievements [6]. Moon has shown positive award-related changes in terms of children's health behaviours, and that the awarded schools have more health promoting culture and organization [7].

The outbreak of Severe Acute Respiratory Syndrome (SARS) has highlighted the importance of maintaining a healthy and hygienic environment as one of the effective public health measures to combat infectious diseases, but the major challenge is that some of the most important public health measures are to be taken outside the health sector [8]. Schools have become an important setting to build up the skills and capacity for students, parents and the wider community to combat the challenges of outbreak of communicable diseases [9]. Rosen et al has demonstrated that a hygiene program can produce a sustained increase in hand washing rates among toilet-trained preschool children [10]. Hand washing has been shown to be an effective means to prevent spread of infection [11]. These findings illuminate the potential of schools as promising venues for promoting hygiene and health in prevention of infectious diseases.

The Centre for Health Education and Health Promotion, The Chinese University of Hong Kong (CHEP) launched the first territory-wide "Healthy Schools Award Scheme" (HSA) in 2001 [12]. It was modeled on the World Health Organization (WHO) Regional Office of the Western Pacific Health Promoting School (HPS) framework covering six key areas (health policy, physical and social environments, community relationships, personal health skills and health services) designed to assist schools in addressing particular health issues strategically [13-16]. The scheme has developed a set of indicators and guidelines to meet the local needs. Each key area has a number of components with targets for the schools to achieve [1315]. The participating schools attended training programme on development planning at the start of the project helping them to appreciate the breadth of the scheme and consolidate their thoughts for appropriate action. The HSA project team met with the co-ordinating group of the school to identify and prioritize four to five areas of potential action. The project team visited the school at least once per term to offer advice and support and obtained detailed information on each of the activities for evaluation. The amount of support given varied from area to area according to the needs of the schools and the summary of health promoting activities have been described in the special issues [17-19].

The process of selection of indicators for the scheme and also the accreditation process have incorporated advice and validation by a number of local and international experts in the field $[15,20]$. A practical manual with detailed guidelines was developed for participating schools $[13,14]$. "Personal health skills" is one key component, where basic knowledge and skills in hygienic measures and prevention of communicable diseases are incorporated in a variety of school activities like classroom teaching, interactive learning initiatives, community-wide health promoting campaigns, and teaching staffs are equipped with relevant training and resources $[9,13-15,20]$.

While process evaluations showed that health promoting activities had been successfully implemented in schools, the evaluation of the effectiveness of HPS reported a wide range of outcomes [21-23]. This survey explores the differences in student perceptions, knowledge and health behaviours between schools that have adopted the HPS framework and those non-HPS. The Education Authority in Hong Kong recommended using the concept of HPS to improve school health and hygiene [24,25] with the hypothesis that children in HPS schools more likely to perceive that their school is a healthier place. The result would have significant impact on the future planning, implementation and substantiation of HPS in different parts of the World as the problem of emerging infectious diseases becomes a global issue.

\section{Method \\ Subjects and sampling}

A cross-sectional study was conducted in two categories of schools, namely HSA and non-HPS by multi-stage random sampling.

At the time of the study there were 24 primary and $17 \mathrm{sec}$ ondary schools that had obtained awards for HPS. For comparison between the two categories of schools, assuming the odds ratio of 2 with the non-HPS school having prevalence of $30 \%$ of parents participated in school cleaning activities, a sample size of 240 were needed for each group $\left(\mathrm{N}=\left\{1.28 \times\left[\mathrm{P}_{1} \times\left(1-\mathrm{P}_{1}\right)+\mathrm{P}_{2} \times(1\right.\right.\right.$ $\left.\left.\left.\mathrm{P}_{2}\right)\right]^{-2}+1.96 \times\left[2 \mathrm{P}_{0}\left(1-\mathrm{P}_{0}\right)\right]^{-2}\right\}^{2} /\left(\mathrm{P}_{2}-\mathrm{P}_{1}\right)^{2} \mathrm{P} 1=$ proportion 
of control, $\left.\mathrm{P}_{2}=\mathrm{P}_{1} \times \mathrm{OR} / 1+\mathrm{P}_{1}(\mathrm{OR}-1) ; \mathrm{P}_{0}=\mathrm{P}_{1}+\mathrm{P}_{2} / 2\right)[26]$. Random sampling method was used to target at least 500 students from primary and secondary schools in HSA category (double the required numbers) and 750 non-HPS category (triple the required numbers) from primary and secondary schools. Schools were randomly selected from each category respectively and letters of invitation were sent out in lots of tens to the selected schools until the targeted numbers of students had been reached.

The first stage consisted of random selection by category and the selected schools were invited by letters. In the second stage, we stratified the participating schools by grades, with samples from Primary 4 (P4) to Primary 6 (P6) (aged around 10-12) and Secondary 1 (S1) to Secondary 3 (S3) (aged around 13-15). The third stage was a systematic random sampling, where one class of students was randomly selected from each grade to participate in the survey.

\section{Measures}

The questionnaire testing health-related knowledge and hygienic practice was devised by the research team in CHEP based on evaluation tools used by Hong Kong HSA $[12,15,20]$ and the content was further validated by two medical doctors with postgraduate degrees in Public Health. Health-related knowledge was reported as a score for each student, and other outcome measures adopt an ordinal scale (e.g. "very clean", "clean", "not quite", "not clean"), which was then grouped into binary variables (e.g. "very clean" and "clean" as a group vs. those not) for statistical analysis. The complete set of questionnaire had been pilot tested in a primary school for face-validity and reliability with good results. The items are well understood. The 2-week test-retest reliability has shown all the items with kappa statistic 0.7 or higher.

The following aspects were examined in the survey:

- Demographic data

- Personal hygienic practices at school, home and public areas

- Students' perception of school health policy

- Community participation by schools

- School physical environment

- Students' knowledge on health and hygiene

- Personal health habits

\section{Data collection}

Data collection was done between May and June of 2005. For the HSA group, 510 students and 789 students were sampled from 5 primary schools and 7 secondary schools respectively. For the 'Non-HPS' group, 676 students and 725 students were sampled from 8 primary schools and 7 secondary schools respectively. The response rate from students of the participating schools was nearly $100 \%$ for all the schools (except those absent from schools). Letters were sent out to students and parents to obtain consent for participation on voluntarily basis. The questionnaires were collected without identifiers to ensure confidentiality. It took about 30 minutes to complete the questionnaire. No refusal was noted from parents and students. The study has been approved by Survey and Behaviour Research Ethics Committee of the Chinese University of Hong Kong

\section{Data analysis}

Proportions of students with particular behaviours and perception of school environment were tabulated. Chi square statistics was used to analyse the difference in proportions between HSA and non-HPS. For knowledge, scores were calculated for each category and t test statistics was used to analyse the difference.

\section{Results}

\section{Demographic Profiles}

The sample consisted of approximately equal gender distribution (see Table 1). The majority of students (around $79 \%$ ) were born in Hong Kong, with a minority born in Mainland (21\%) or other countries. The mean age of the participants from secondary schools was 14.46 (S.D. \pm

Table I: Grade and Gender of the respondents by school category

\begin{tabular}{lll}
\hline Primary schools & HSA (n = 5 I 0) & Non-HPS (n = 676) \\
\hline P.4 & $165(32.4 \%)$ & $228(33.7 \%)$ \\
P.5 & $168(32.9 \%)$ & $217(32.1 \%)$ \\
P.6 & $177(34.7 \%)$ & $231(34.2 \%)$ \\
Boy & $242(47.6 \%)$ & $359(53.3 \%)$ \\
Girl & $266(52.4 \%)$ & $315(46.7 \%)$ \\
\hline Secondary schools & HSA (n = 789) & Non-HPS (n = 725) \\
\hline S.I & & \\
S.2 & $260(33.0 \%)$ & $217(29.9 \%)$ \\
S.3 & $273(34.6 \%)$ & $260(35.9 \%)$ \\
Boy & $256(32.4 \%)$ & $248(34.2 \%)$ \\
Girl & $367(46.9 \%)$ & $308(42.6 \%)$ \\
& $415(53.1 \%)$ & $415(57.4 \%)$ \\
\hline
\end{tabular}

(Note: A minority of the students missed filling in their demographic data)

HSA: awarded schools in the HSA Scheme

Non-HPS schools are those not in the HSA scheme and whose teachers did not attend any training in HPS or diplomas 
1.33 ) and 14.51 (S.D. \pm 1.25 ) year-old in HSA and nonHPS schools respectively. The mean age of the participants from primary schools was 11.15 (S.D. \pm 0.95 ) and 11.54 (S.D. \pm 1.56$)$ year-old in HSA and non-HPS schools respectively.

\section{Personal hygienic practices and skills at school, home and public areas}

Handwashing practices

In terms of hand washing habits after toileting, only small difference was detected as this is something that has been enmeshed into the children's everyday life especially since the SARS epidemic.

There was a significant difference between HSA schools and non-HPS schools in teachers reminding students to wash their hand before meals (Table 2). Also higher proportion of secondary school students washed hands with their own initiatives before meals although the reverse was observed amongst primary students (Table 2)

\section{Tooth Brushing and Bathing}

Table 2 shows that in primary schools, higher proportion of students from HSA schools (28.2\%) always brushed their teeth after meal than the non-HPS category (19.7\%) with statistical significance $(\mathrm{p}=0.001)$ (Table 2$)$.

The majority of students had a bath everyday but HSA primary school students reported a significantly higher proportion than non-HPS schools (99.6\% vs. $98.1 \%$, p = 0.02 ; table 2).

\section{Correct self care with fever}

Higher proportion of students from HSA schools in both primary and secondary schools $(80.9 \%$ vs $75.2 \%$ and $64.0 \%$ vs $58.0 \%$ respectively) coped correctly when they had fever with statistical significance ( $\mathrm{p}=0.02$; Table 2$)$.

\section{School health policy}

There was no significant difference amongst primary school children encouraged to wear face mask or staying away from school if they had symptoms of Upper Respiratory Tract Infection (URTI). However difference was observed in mask wearing between the HSA and non-HPS secondary schools ( $86.8 \%$ vs $80.4 \%$ ) and in staying away from schools if students had URTI symptoms (93.6\% vs $90.1 \%)$ with statistical significance $(\mathrm{p}=0.001$ and $\mathrm{p}=$ 0.01 respectively, Table 3 ).

The Hygiene Charter, launched in 2003 as a community initiative supported by various business and industry sectors to pledge their long-term commitment to fight against the challenges posed by SARS and create a healthy and hygienic environment for Hong Kong [23]. Its website is of high reference value for schools. In secondary schools, higher proportion of students from the HSA group reported that teachers had mentioned the Hygiene Charter website (28\% vs $15.3 \%$; $<0.001$ ) (Table 3 ).

\section{Community participation by schools}

In secondary schools, higher proportion of students from the HSA group (23.6\% vs $19.2 \%$ ) felt that their schools joined community cleaning with statistical significance $(\mathrm{p}$ $=0.04$ ) (Table 4 ). The organization of activities to promote health and hygiene was also reported by higher proportion of students from the HSA than the non-HPS

Table 2: Personal hygienic practices and skills at school, home and public areas

\begin{tabular}{|c|c|c|c|}
\hline & HSA & Non-HPS & P value \\
\hline \multicolumn{4}{|c|}{ I. Handwashing Before meal always } \\
\hline Primary & $60.4 \%(307 / 508)$ & $66.1 \%(446 / 675)$ & 0.05 \\
\hline Secondary & $46.6 \%(366 / 785)$ & $40.1 \%(291 / 725)$ & 0.01 \\
\hline \multicolumn{4}{|c|}{ 2. Handwashing after toileting always } \\
\hline Primary & $94.9 \%(482 / 508)$ & $92.6 \%(624 / 674)$ & 0.11 \\
\hline Secondary & $93.6 \%(737 / 787)$ & $93.2 \%(676 / 725)$ & 0.75 \\
\hline \multicolumn{4}{|c|}{ 3. Teachers reminded students to wash hands before meal } \\
\hline Primary & $65.2 \%(332 / 509)$ & $75.9 \%(491 / 647)$ & $<0.001$ \\
\hline Secondary & $31.9 \%(251 / 786)$ & $8.3 \%(60 / 722)$ & $<0.001$ \\
\hline \multicolumn{4}{|c|}{ 4. Brush teeth after meal always } \\
\hline Primary & $28.2 \%(143 / 507)$ & $19.7 \%(133 / 675)$ & 0.001 \\
\hline Secondary & $10.7 \%(84 / 783)$ & $12.4 \%(90 / 724)$ & 0.30 \\
\hline \multicolumn{4}{|c|}{ 5. Bath everyday } \\
\hline Primary & $99.6 \%(506 / 508)$ & $98.1 \%(663 / 676)$ & 0.02 \\
\hline Secondary & $98.9 \%(777 / 786)$ & $98.3 \%(710 / 722)$ & 0.39 \\
\hline \multicolumn{4}{|c|}{ 6. correct coping when fever } \\
\hline Primary & $80.9 \%(4 \mid 2 / 509)$ & $75.2 \%(507 / 674)$ & 0.02 \\
\hline Secondary & $64.0 \%(497 / 777)$ & $58.0 \%(4|8 / 72|)$ & 0.02 \\
\hline
\end{tabular}


Table 3: Students' perception of school health policy

\begin{tabular}{|c|c|c|c|}
\hline & HSA & Non-HPS & p value \\
\hline \multicolumn{4}{|c|}{ I. Schools encourage face mask wearing when URTI } \\
\hline Primary & $88.4 \%(450 / 509)$ & $88.0 \%(595 / 676)$ & 0.84 \\
\hline Secondary & $86.8 \%(681 / 785)$ & $80.4 \%(582 / 724)$ & 0.001 \\
\hline \multicolumn{4}{|c|}{ 2. Schools tell student to stay away from school when URTI } \\
\hline Primary & $96.7 \%(49 \mid / 508)$ & $95.5 \%(644 / 674)$ & 0.34 \\
\hline Secondary & $93.6 \%(737 / 787)$ & $90.1 \%(652 / 724)$ & 0.01 \\
\hline \multicolumn{4}{|c|}{ 3. Schools mention hygiene charter website } \\
\hline Primary & $30.6 \%(156 / 509)$ & $30.8 \%(207 / 67 \mid)$ & 0.94 \\
\hline Secondary & $28.1 \%(221 / 786)$ & $15.3 \%(|| \mid / 724)$ & $<0.001$ \\
\hline
\end{tabular}

group in both primary (60.9\% vs. $56.7 \%)$ and secondary schools $(53.7 \%$ vs. $37.0 \%$, p < 0.001) (Table 4). Significantly higher proportion of parents from the HSA group joined school cleaning activities in both primary and secondary schools $(42.7 \%$ vs. $31.9 \%$; p $<0.001$, and $20.3 \%$ vs. $8.6 \% ; \mathrm{p}<0.001$ respectively) (Table 4 ).

\section{School physical environment}

Primary school students in the HSA group had higher proportion choosing "very clean" or "clean" for playground hygiene $(72.2 \%)$ as compared to $61.6 \%$ in the non-HPS group ( $\mathrm{p}<0.001$; Table 4). For classroom $73.6 \%$ of students from HSA schools chose "very clean" or "clean" compared with $68.5 \%$ in non-HPS schools $(\mathrm{p}=0.058)$ (Table 4). Secondary school students in HSA group had higher proportion choosing "very clean" or "clean" for stairs $(68.7 \%$ vs $63.3 \% \mathrm{p}=0.03)$ and tuck shop $(60.2 \%$ vs $53.3 \% \mathrm{p}=0.01)($ Table 4$)$.

More HSA primary $(75.0 \%$ vs. $64.4 \% ; \mathrm{p}<0.001)$ and secondary $(52.5 \%$ vs. $34.2 \%, \mathrm{p}<0.001)$ students perceived their schools having assigned prefects to monitor or help

Table 4: Community Participation and Physical Environment

\begin{tabular}{|c|c|c|c|}
\hline & HSA & Non-HPS & p value \\
\hline \multicolumn{4}{|c|}{ I. Schools Join community cleaning } \\
\hline Primary & $43.9 \%(219 / 509)$ & $43.1 \%(291 / 675)$ & 0.97 \\
\hline Secondary & $23.6 \%(186 / 787)$ & $19.2 \%(139 / 725)$ & 0.04 \\
\hline \multicolumn{4}{|c|}{ 2. school organize health promoting activities } \\
\hline Primary & $60.9 \%(310 / 509)$ & $56.7 \%(382 / 674)$ & 0.14 \\
\hline Secondary & $53.7 \%(422 / 786)$ & $37.0 \%(268 / 724)$ & $<0.001$ \\
\hline \multicolumn{4}{|c|}{ 3. Parents join school cleaning activities } \\
\hline Primary & $42.7 \%(217 / 508)$ & $31.9 \%(215 / 675)$ & $<0.001$ \\
\hline Secondary & $20.3 \%(160 / 787)$ & $8.6 \%(62 / 723)$ & $<0.001$ \\
\hline \multicolumn{4}{|c|}{ 4. Playgrounds perceived as very clean or clean } \\
\hline Primary & $72.2 \%(366 / 507)$ & $61.6 \%(415 / 674)$ & $<0.001$ \\
\hline Secondary & $56.5 \%(444 / 786)$ & $53.5 \%(386 / 721)$ & 0.25 \\
\hline \multicolumn{4}{|c|}{ 5. Classrooms perceived as very clean or clean } \\
\hline Primary & $73.6 \%(374 / 508)$ & $68.5 \%(462 / 674)$ & 0.058 \\
\hline Secondary & $51.3 \%(404 / 787)$ & $52.5 \%(380 / 724)$ & 0.65 \\
\hline \multicolumn{4}{|c|}{ 6. Toilets perceived as very clean or clean } \\
\hline Primary & $34.7 \%(176 / 507)$ & $38.5 \%(259 / 673)$ & 0.18 \\
\hline Secondary & $29.7 \%(233 / 784)$ & $27.6 \%(\mid 99 / 721)$ & 0.36 \\
\hline \multicolumn{4}{|c|}{ 7. Staircases perceived as very clean or clean } \\
\hline Primary & $77.2 \%(392 / 508)$ & $76.2 \%(5 / 3 / 673)$ & 0.71 \\
\hline Secondary & $68.7 \%(540 / 786)$ & $63.3 \%(457 / 722)$ & 0.03 \\
\hline \multicolumn{4}{|c|}{ 8. Tuckshop perceived as very clean or clean } \\
\hline Primary & $84.1 \%(428 / 509)$ & $81.4 \%(407 / 500)$ & 0.26 \\
\hline Secondary & $60.2 \%(47 I / 783)$ & $53.3 \%(313 / 587)$ & 0.01 \\
\hline \multicolumn{4}{|c|}{ 9. Prefect for cleaning } \\
\hline Primary & $75.0 \%(382 / 509)$ & $64.4 \%(435 / 675)$ & $<0.001$ \\
\hline Secondary & $52.5 \%(4 \mid 2 / 785)$ & $34.2 \%(247 / 723)$ & $<0.001$ \\
\hline \multicolumn{4}{|c|}{ 10. Prefects for environment Protection } \\
\hline Primary & $63.1 \%(321 / 509)$ & $59.7 \%(403 / 675)$ & 0.24 \\
\hline Secondary & $49.4 \%(388 / 786)$ & $23.7 \%(171 / 723)$ & $<0.001$ \\
\hline
\end{tabular}


with cleaning with statistical significance. More HSA primary school students reported having prefects for environmental protection ( $63.1 \%$ vs. $59.7 \%$ ), and similarly in secondary schools (49.4\% vs. $23.7 \%)$ with statistical significance $(\mathrm{p}<0.001)$ (Table 4$)$.

\section{Literacy on health and hygiene}

In most areas of health-related knowledge, it was found that the majority of students in both groups have ever acquired related knowledge. There was no significant difference amongst the secondary school students between the HSA and non-HPS groups in the overall scores for both categories ( 12.40 vs. $12.41 ; \mathrm{p}=0.95$ ). However in primary schools, the difference in overall scores between the two groups was found to be statistically significant (12.13 vs 11.58; $\mathrm{p}<0.001$ ) (Table 5).

Higher proportion of primary school students in HSA group had chosen "very much" or "quite a lot" in getting health information from student health ambassadors (32.8\% vs $17.7 \%$; p < 0.001 ), media ( $46.5 \%$ vs. $43.2 \%$; $=0.002)$, and pamphlets (52.3\% vs $46.2 \% \mathrm{p}=0.04)$ with statistical significance (Table 5). For secondary schools, it was also found that higher proportion of students in HSA group had chosen "very much" or "quite a lot" in getting health information from student ambassadors $(19.0 \%$ vs $10.5 \%$; $\mathrm{p}<0.001)$ and pamphlets $(36.5 \%$ vs $27.3 \% \mathrm{p}<$ 0.001 ) with statistical significance (Table 5).

\section{Discussion}

On the whole, students from the HSA group showed positive outcomes in most aspects of health and hygiene particularly the hygiene practice of students and actions taken by schools. It is not unexpected as HPS embodies a holistic, whole school approach to personal and community health promotion [27]. The Hong Kong Healthy Schools Award scheme builds on the concept of HPS which helps to create a supportive environment for healthy development in schools, home and the community [6]. Studies have shown that students from schools which had comprehensively embraced the HPS concept as indicated by attaining the 'Healthy School Award', were better, in terms of health and educational outcomes, than students from schools that did not reach the standard of the award $[28,29]$.

St Leger and Nutbeam [30] stated that HPS contributes to four school related outcomes. They are: (a) lifelong learning; (b) competencies and behaviors; (c) specific cognate knowledge and skills; and (d) self attributes. Therefore HSA schools would confer a great impact on school health and hygiene, in addition to students' health habits, as was demonstrated in the present study.

Positive changes were observed in certain aspects of school health and hygiene particularly in getting relevant health information from various reliable sources amongst secondary students in the HSA group. Previous studies have indicated the potential for a settings approach to school health, like the Hong Kong HSA scheme (HKHSA),

Table 5: Literacy on health and hygiene

\begin{tabular}{|c|c|c|c|}
\hline & HSA & Non-HPS & p value \\
\hline \multicolumn{4}{|c|}{ I. Health knowledge scores (mean \pm SD) } \\
\hline Primary & $12.13+2.45$ & $11.58+2.82$ & $<0.001$ \\
\hline Secondary & $12.40+2.44$ & $12.4 \mid+2.69$ & 0.95 \\
\hline \multicolumn{4}{|c|}{ 2. get health info from teachers (very much or quite a lot) } \\
\hline Primary & $86.7 \%(44 I / 509)$ & $84.2 \%(568 / 674)$ & 0.25 \\
\hline Secondary & $69.4 \%(546 / 786)$ & $68.7 \%(498 / 725)$ & 0.74 \\
\hline \multicolumn{4}{|c|}{ 3. Get health info from classmates (very much or quite a lot) } \\
\hline Primary & $39.4 \%(200 / 508)$ & $38.8 \%(260 / 67 I)$ & 0.83 \\
\hline Secondary & $28.4 \%(223 / 786)$ & $26.6 \%(193 / 725)$ & 0.45 \\
\hline \multicolumn{4}{|c|}{ 4. Get health Information from ambassador (very much or quite a lot) } \\
\hline Primary & $32.8 \%(165 / 503)$ & $17.7 \%(119 / 673)$ & $<0.001$ \\
\hline Secondary & $19.0 \%(149 / 785)$ & $10.5 \%(76 / 720)$ & $<0.001$ \\
\hline \multicolumn{4}{|c|}{ 5. Get health info from media (very much or quite a lot) } \\
\hline Primary & $46.5 \%(236 / 507)$ & $43.2 \%(29 I / 673)$ & 0.002 \\
\hline Secondary & $48.5 \%(380 / 783)$ & $49.8 \%(360 / 724)$ & 0.64 \\
\hline \multicolumn{4}{|c|}{ 6. Get health info from pamphlets (very much or quite a lot) } \\
\hline Primary & $52.3 \%(265 / 507)$ & $46.2 \%(311 / 674)$ & 0.04 \\
\hline Secondary & $36.5 \%(287 / 786)$ & $27.3 \%(197 / 722)$ & $<0.001$ \\
\hline \multicolumn{4}{|c|}{ 7. Get health info from website (very much or quite a lot) } \\
\hline Primary & $42.3 \%(215 / 508)$ & $44.9 \%(303 / 674)$ & 0.37 \\
\hline Secondary & $20.3 \%(160 / 786)$ & $19.7 \%(143 / 724)$ & 0.77 \\
\hline
\end{tabular}


to provide a promising strategic framework allowing health literacy outcome to be achieved [2,31]. This group of schools also established actions to promote better health and hygiene as the schools participated in community cleaning activities and the parents also supported the schools in cleaning activities. This study reveals that these HSA schools have also organized more health promotion activities. This moves to a more collaborative communitybased approach for students to learn, as opposed to a teacher-dominated school hierarchy [2], is one of the strategies to achieve critical health literacy [1].

School-based implementation of HPS was shown to be associated with significant greater levels of positive personal health habits. Although this study is cross-sectional and no cause and effect can be established, it would still highlight how HKHSA might exert its influence individually as well as the school level. Although some of the significant findings of this study is marginal, HPS has only been implemented over a short duration in Hong Kong. HPS programme has been shown to be effective if the intervention is intensive over a long duration [32]. Recent evidence suggests that the way the school is lead and managed, students' experiences in participating and taking responsibility for shaping policies, practices and procedures, teachers-students relationship and school engagement with its local community including parents actually builds many protective factors for health and minimises risk taking behaviour [32-35]. Many of these gains have occurred without a specific health 'intervention'. It appears that a whole school approach to build and maintain a caring school social environment might be the most effective way in achieving good health outcomes.

However putting the HPS in practice takes considerable amount of time and efforts and effective implementation requires commitment and support of various schools' stakeholders. The success of HPS depends heavily on teachers' training like Health Education diploma programmes, which could introduce the concepts to the teachers so they can start making improvements on some fundamental areas of school health and hygiene [36,37]. With time and more teachers being exposed to the concept, teachers would then gradually develop their schools to become HPS. The primary school students in HSA group have shown to have better knowledge scores. Although the difference in knowledge scores between two groups is not statistically significant in secondary schools, it is well known fact that there are other factors in adolescents leading to behaviour and practice change apart from knowledge and belief.

There are some limitations of this study. Schools with more health conscious students or their families might be more willing to adopt HPS. The study design might not be able to control for that history which would have some threat to internal validity. It is a cross sectional survey so it might not capture the longer-term impact of HPS on health behaviours of students due to the different components of HSA interventions. The differences observed might or might not be due to the effect of intervention and future longitudinal studies are needed to ascertain the causal effect fostered by HSA. Furthermore, some outcomes were reported by students only and may involve recall in the past few months, and the effect of multiple comparisons should be taken into account when interpreting statistical significance. In addition, we assumed that randomized sampling minimized the heterogeneity of different clusters and demographic characteristics among schools, which have not been controlled for. This study did not comprehensively assess all the outcomes of HPS as there are different types of outcomes which require different methods of data collection. [14] A descriptive design was adopted instead of randomised control study design because one cannot randomly select schools from all schools in Hong Kong as intervention group since schools need to comply with the standards of HPS to make it an effective programme.

\section{Conclusion}

The study has demonstrated that schools involved in HPS activities through the HSA scheme in Hong Kong had more favorable hygiene practice of students, school environment and atmosphere in health and hygienic practice. Although all schools in Hong Kong have health elements in the curriculum, the primary objective of HPS is to modify the behaviours of school students rather than information giving, so more positive changes are observed in those schools that participated and met the standards in the HSA scheme. These findings highlight the potential of HPS in equipping schools to handle public health crisis such as SARS [9]. Therefore it is highly recommended that schools would further enhance the practice of HPS to empower their students, staffs and parents to enhance preparedness of possible future epidemics of communicable diseases. [38].

\section{Competing interests}

The author(s) declare that they have no competing interests.

\section{Authors' contributions}

Contributions by different authors are as followings-:

- AL and MW were responsible for conception and design, analysis and interpretation of data, drafting and revising the manuscript and gave the final approval 
- FC, HY and VK were responsible for conception and design, analysis and interpretation of data, and gave the final approval

- JM was responsible for conception and design, revising the manuscript and gave the final approval

\section{References}

I. Nutbeam D: Health literacy as a public health goal: a challenge for contemporary health education and communication strategies into the $\left.2\right|^{\text {st }}$ century. Health Promotion International 2000, 15(3):259-267.

2. St Leger L: Schools, health literacy and public: possibilities and challenges. Health Promotion International 200I, I 6(2): 197-205.

3. Nutbeam D: The health promoting school: organisation and policy development in Welsh secondary schools. Health Education 1987, 46:109-1I5.

4. Young I: Health promoting schools: healthy eating policies in schools - an evaluation of the effects on pupils' knowledge, attitudes and behaviour. Health Education Journal 1993, 52:I.

5. Parsons $C$, Stears $D$, Thomas $C$ : The health promoting school in Europe: Conceptualising and evaluating the change. Health Education Journal 1996, 55:3II-32I.

6. Rogers E, Moon AV, Mullee MA, Speller VM, Roderick PJ: Developing the "health-promoting school" - a national survey of healthy school awards. Public Health 1998, I | 2:37-40.

7. Moon AM, Mullee MA, Rogers L, Thompson RL, Speller V, Roderick $P$ : Helping schools to become health-promoting environments - an evaluation of the Wessex Healthy Schools Award. Health Promotion International 1999, 14: I I I-122.

8. Lee A, Abdullah ASM: Severe Acute Respiratory Syndrome: Challenge for Public Health Practice in Hong Kong. Journal of Epidemiology and Community Health 2003, 57:655-658.

9. Lee A, Cheng F, Yuen H, Ho M: Healthy Schools Support Group, How would schools step up public health measure to control spread of SARS? Journal of Epidemiology and Community Health 2003, 57:945-949.

10. Rosen L, Manor O, Engelhard D, Brody D, Rosen B, Peleg H, Meir M, Zucker D: Can a handwashing intervention make a difference? Results from a randomized controlled trial in Jerusalem preschools. Preventive Medicine 2006, 42(I):27-32.

11. Larson EA: A causal link between handwashing and risk of infection? Examination of the evidence. Infect Control Hosp Epidemiol 1988, 9:28-36.

12. Lee A: Helping schools to promote healthy educational environments as new initiatives for school based management: the Hong Kong Healthy Schools Award Scheme. Promotion and Education 2002, SI:29-32.

13. Lee A, Ho M, Leung TCY, et al.: Development of indicators and guidelines for the Hong Kong Healthy Schools Award Scheme. Journal of Primary Care and Health Promotion 2004, I(I):4-9.

14. Lee A, Editor: Hong Kong Healthy Schools Award Scheme: Practical Guide to the Health Promoting School. Full manual and Abridged version (74 pages) Centre for Health Education and Health Promotion, School of Public Health, The Chinese University of Hong Kong; 2003.

15. Lee A, Cheng FFK, St Leger L: Evaluating health promoting schools in Hong Kong: the development of a framework. Health Promotion International 2005, 20(2): I77-186.

16. WHO Regional Office for the Western Pacific: Health-Promoting Schools Series 5: Regional guidelines. Development of health-promoting schools - A framework for action 1996. WHO/WPRO

17. Centre for Health Education and Health Promotion: The Hong Kong Healthy Schools Award Scheme Special Issue 2005 Hong Kong: Centre for Health Education and Health Promotion, The Chinese University of Hong Kong; 2005. ISBN 988-9834I-9-7

18. Centre for Health Education and Health Promotion: The Hong Kong Healthy Schools Award Scheme Special Issue 2004 Hong Kong: Centre for Health Education and Health Promotion, The Chinese University of Hong Kong; 2004. ISBN: 962-86985-0-8

19. Centre for Health Education and Health Promotion: The Hong Kong Healthy Schools Award Scheme Special Issue 2003 Hong Kong: Centre for Health Education and Health Promotion, The Chinese University of Hong Kong; 2003.
20. Lee A, St Leger L, Moon A: Evaluating health promotion in schools: a case study of design, implementation and results from the Hong Kong Healthy Schools Award Scheme. Promotion \& Education 2005, 7(3-4): |23-130.

21. Simovska V: Student participation: a democratic education perspective - experience from the health-promoting schools in Macedonia. Health Education Research 2004, 19(2): 198-207.

22. Mukoma W, Flisher AJ: Evaluations of health-promoting schools: a review of nine studies. Health Promotion International 2004, 19(3):357-368.

23. Database of Abstracts of Reviews of Effectiveness: Health Promoting schools and health promotion in schools: two systematic reviews (Structured abstract) Centre for Review and Dissemination; 2006.

24. Hong Kong SAR Government: Team Clean Report of Hong Kong Special Administrative Government 2003.

25. Lee A, Chan KM: Hygiene Charter: Laying down the spirit of Healthy City. Gallery Paper. Journal of Epidemiology and Community Health 2005, 59:30.

26. Kirkwood BR: Essentials of Medical Statistics Blackwell Scientific Publications, Oxford; 1998.

27. Parsons C, Stears D, Thomas C: The health promoting school in Europe: Conceptualising and evaluating the change. Health Education Journal 1996, 55:3। I-32I.

28. Moon A: Does a healthy school award scheme make a difference? The evaluation of the Wessex Healthy Schools Award, unpublished PhD Thesis, Department of Public Health Medicine, University of Southampton, United Kingdom 1999.

29. Lee A, Cheng F, Fung Y, St Leger L: Can Health Promoting Schools contribute to the better health and well being of young people: Hong Kong experience? Journal of Epidemiology and Community Health 2006, 60:530-536.

30. St Leger L, Nutbeam D: A model for mapping linkages between health and education agencies to improve school health. Journal of School Health 2000, 70:45-50.

31. Lister-Sharp D, Chapman S, Stewart-Brown S, Sowden A: Health promoting schools and health promotion in schools: two systematic reviews. Health Technology Assessment 1999, 3: I-207.

32. Stewart-Brown S: What is the evidence on school health promotion in improving school health or preventing disease and specifically what is the effectiveness of the health promoting schools approach? Copenhagen: World Health Organization; 2006.

33. Patton G, Bond L, Carlin J, Thomas L, Butler H, Glover S, Catalano R, Bowes G: Promoting social inclusion in schools: A group-randomized trial on student health risk behaviour and wellbeing. American Journal of Public Health 2000, 96:9.

34. Blum R, McNeely C, Rinehart P: Improving the odds: The untapped power of schools to improve the health of teens Center for Adolescent Health and Development, University of Minnesota; 2002.

35. St Leger L, Kobe LJ, Lee A, McCall D, Young I: School Health: - Achievements, Challenges and Priorities Edited by: McQueen D, Jones C. Global Perspective on Health Promotion Effectiveness. Springer, New York, USA; 2007.

36. Hausman A, Ruzek SB: Implementation of comprehensive school health education in elementary schools: focus on teacher concerns. Journal of School Health 1995, 65(3):8I-86.

37. Lee A, Lee SH, Tsang KK, To CY: A comprehensive "Healthy Schools Programme" to promote school health: The Hong Kong experience in joining the efforts of Health and Education Sectors. Journal of Epidemiology and Community Health 2003, 57:174-177.

38. Lee A: Will SARS epidemics recur? Journal of Epidemiology and Community Health 2003, 57:770.

\section{Pre-publication history}

The pre-publication history for this paper can be accessed here:

http://www.biomedcentral.com/1471-2458/8/42/prepub 